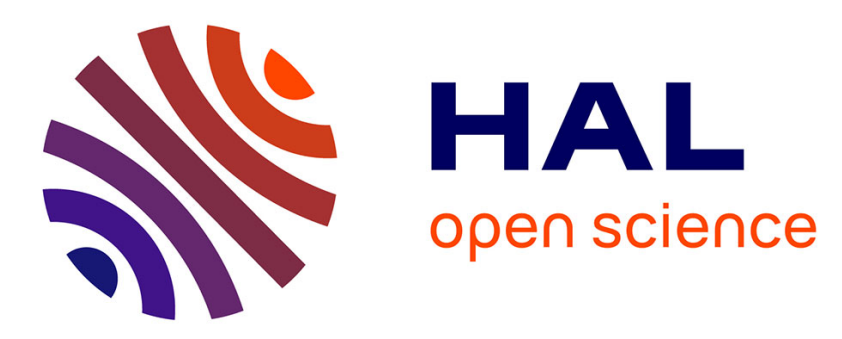

\title{
Failure prediction for ceramic dies in the hot-forging process using FEM simulation
}

\author{
Frédéric Deschaux-Beaume, Fabrice Schmidt, N. Fréty, J.C. Boyer, C.
}

Levaillant

\section{- To cite this version:}

Frédéric Deschaux-Beaume, Fabrice Schmidt, N. Fréty, J.C. Boyer, C. Levaillant. Failure prediction for ceramic dies in the hot-forging process using FEM simulation. Journal of Materials Processing Technology, 1998, 75 (1-3), pp.100-110. 10.1016/S0924-0136(97)00298-7 . hal-02051047

\section{HAL Id: hal-02051047 https://imt-mines-albi.hal.science/hal-02051047}

Submitted on 27 Feb 2019

HAL is a multi-disciplinary open access archive for the deposit and dissemination of scientific research documents, whether they are published or not. The documents may come from teaching and research institutions in France or abroad, or from public or private research centers.
L'archive ouverte pluridisciplinaire HAL, est destinée au dépôt et à la diffusion de documents scientifiques de niveau recherche, publiés ou non, émanant des établissements d'enseignement et de recherche français ou étrangers, des laboratoires publics ou privés. 


\title{
Failure prediction for ceramic dies in the hot-forging process using FEM simulation
}

\author{
F. Deschaux-Beaume ${ }^{\mathrm{a}, *}$, F. Schmidt ${ }^{\mathrm{a}}$, N. Fréty ${ }^{\mathrm{a}}$, J.C. Boyer $^{\mathrm{b}}$, C. Levaillant ${ }^{\mathrm{a}}$ \\ a Ecole des Mines d'Albi, 81013 Albi, France \\ b INSA-Lyon, 69621 Villeurbanne, France
}

\begin{abstract}
A fracture prediction criterion for brittle materials has been introduced in the POLLUX finite-element code, in order to predict the risk of rupture of ceramic tools during a forging operation. The POLLUX code, developed by INSA (Lyon) especially to simulate forging operations, is presented. The fracture model is based on the weakest link theory and Weibull analysis. Two different criteria were chosen in order to characterise the stress state, considering the tensile normal stresses. Comparison between the simulation results and the analytical calculations, in a simple compression case, enables the validation of the numerical model. Applications are presented, in which the design of ceramic forging tools is realized using the failure prediction software. A run-strategy of the program is proposed in order to improve the design of the forging tools.
\end{abstract}

Keywords: Finite-element method; Fracture prediction; Ceramics; Forging

\section{Introduction}

In the aircraft industry, the development of engines with higher performance, has led to the introduction of new titanium and nickel base materials, requiring forming techniques that are quite different from those used for more conventional products. The isothermal forging of superplastic materials has appeared as the most appropriate forming process of some titanium alloys and nickel-base superalloys. This technique should allow the production of near-net-shape pieces directly from simple-shape preforms. However, superplastics properties are achieved only at high temperature and low strain-rate for these alloys. For instance, in the case of some nickel-base alloys, superplasticity is reached at temperatures exceeding $1373 \mathrm{~K}$, which means that the material for the forging dies, which will be used at the same temperature as the workpiece, must be heat resistant.

The isothermal forging of advanced alloys has been carried out using metal dies (Mo based), which requires a controlled atmosphere system in order to limit the

* Corresponding author. Fax: + 33563493099 . oxidation of the dies. The use of ceramic dies should be a less expensive way, as the very good oxidation resistance of these materials does not require a controlled atmosphere. Advanced ceramics are also attractive for their physical and mechanical properties, such as their high-temperature strength, their light weight and excellent erosion resistance. However, ceramics are inherently brittle, and the lack of ductility leads to low fracture toughness and large variations in observed fracture strength. The usual design techniques are not suited to ceramic components. The wide variation of material strength is due to the nature and distribution of intrinsic microscopic flaws, that are unavoidably present as a result of material manufacturing operations. Failure in ceramics usually initiates at a single weakest flaw when the local stress reaches a critical value. Statistics and reliability analysis are therefore necessary to predict the failure of brittle material components as a function of service time.

Statistical fracture models, based on the weakest-link theory, have been developed previously. This theory assumes that the structure is analogous to a chain with many links. Each link may have a different limiting strength. When a load is applied to the structure such that the weakest link fails, then the structure fails. 
The first probabilistic approach used to account for the scatter in fracture strength of brittle materials was introduced by Weibull [1]. This model requires statistical parameters to phenomenologically describe the failure response of a material. These statistical parameters, depending on the material characteristics, are usually determined from uniaxially-loaded, simple-geometry samples. In order to predict the material behaviour in other stress states using statistical parameters from uniaxial tests, Weibull proposed the calculation of the risk of rupture considering the maximal tensile principal stress or averaging the tensile normal stress in all directions. However, several investigators have shown that these models could lead to unsafe estimates of failure probability, and another model was introduced, based on the assumption that the principal stresses act independently [2]. In this method, the reliability of a component under multi-axial stresses is the product of the reliability of the individual principal stresses acting independently.

In another model, Batdorf [3] combines the weakestlink theory and linear elastic fracture mechanics. Conventional fracture mechanics analysis assumes that both the size of the critical crack and its orientation respective to the applied load determine the fracture stress. The Batdorf theory includes the calculation of the combined probability of the critical flaw being within a particular size range and being located and oriented so that it may cause fracture. In addition to the statistical parameters determined, as for the Weibull model, by simple uniaxial tests, other parameters characterising the flaw orientation as a function of the stress directions must be calculated. These parameters can be difficult to determine for complex shapes and stress states.

Except in some simple cases, it is necessary, for predicting the risk of failure of ceramics structures with these different models, to use numerical simulation. Some studies have been conducted previously in which brittle fracture models were coupled to finite-element simulations to calculate the failure probability of particular ceramic structures. Berdin [4] introduced both the Weibull and Batdorf models in the post processor of the Zebulon finite-element program, to predict the failure of particular disks under biaxial stresses. The statistical parameters of both models were determined from four point bending tests. In this study, results of the Weibull analysis for fractured disks were in better agreement with the experimental results than those of the Batdorf analysis.

Lamon [5] has also developed a numerical model of statistical fracture analysis of ceramic composites in the FLAG post processor of a commercial finite-element program named MARC. The simulation, based on the Weibull approach and on the properties of different composite constituents, has led to the computation of the distribution of failure probabilities, and the global failure probability of a unit cell, as a function of strain.

However, most of the research in this field has been realised by the National Aeronautics and Space Administration [6-8]. In the late 1970s, NASA initiated the development of the SCARE computerised design program for the reliability evaluation of ceramic structures. SCARE can be coupled to the commercial finite-element programs ANSYS, ABAQUS, NASTRAN and COSMOS, and use the output data from these programs. The Weibull and Batdorf fracture models are present in the code, but simulation results show that the selected criterion has no significant effect on fracture prediction [9]. Nevertheless, the finite-element programs quoted above are not convenient for the simulation of the forging process, where large strains take place in the workpiece.

The objective of this study is to develop a finite-element model in order to be able to predict the failure of ceramic forging dies as a function of the service conditions, in order to improve the design of these components. This numerical model has been introduced into the POLLUX finite-element code, developed previously by INSA Lyon [10]. The Weibull model has been chosen to describe the fracture characteristics of ceramic materials, because the statistical parameters of this model are easy to determine experimentally. Both the maximal principal stress and the principle of independent actions criteria are available in the model. One of the principal characteristics of the program is that the fracture probability of the tools is calculated at each increment. This calculation is run at the same time increment as the stress tensor evaluation. The limit fracture probability, which is chosen by the user, controls the end of the simulation.

\section{Software description}

The finite-element code POLLUX has been developed in order to predict the stress state in the workpiece and the tools during a forging process. The thermo-elasto-visco-plastic formulation is based on a displacement approach. The model uses a separated implicit integration scheme of the constitutive law [10] and is presented in the following sections.

\subsection{Displacement formulation}

\subsubsection{Thermo-elasto-plastic constitutive law}

The Cauchy stress tensor $\bar{\sigma}$ can be deduced from the elastic strain tensor $\overline{\varepsilon^{e}}$, itself deduced from the difference between the total strain $\overline{\varepsilon^{\mathrm{t}}}$, the plastic strain $\overline{\varepsilon^{\mathrm{p}}}$, and the thermal strain $\overline{\varepsilon^{\text {th }}}$, and the elastic behaviour of the material represented by the fourth-order tensor $C$, which can be temperature dependant: 
$\bar{\sigma}=\bar{C}: \overline{\varepsilon^{\mathrm{e}}}=\bar{C}:\left(\overline{\varepsilon^{\mathrm{t}}}-\overline{\varepsilon^{\mathrm{p}}}-\overline{\varepsilon^{\mathrm{th}}}\right)$

\subsubsection{Equilibrium equation for a displacement approach}

Consider a continuum media $V$, with an equilibrium stress field $\bar{\sigma}$ at time $t$, subjected to a loading step for time $\mathrm{d} t$. The displacement field occurring during $\mathrm{d} t$ is the solution of the equilibrium equation applied to the loading increment. A weak formulation with the test function $\vec{w}$ of the equilibrium equation on the continuum media $V$ and the introduction of the elasto-plastic constitutive law give the following equation:

$\int_{V}\left(\bar{C}: \Delta \overline{\varepsilon^{t}}\right): \operatorname{grad} \vec{w} \mathrm{~d} v$

$=\int_{S} \Delta \vec{\Phi} \cdot \vec{w} \mathrm{~d} s+\int_{V}\left(\bar{C}: \Delta \overline{\varepsilon^{\mathrm{p}}}\right): \operatorname{grad} \vec{w} \mathrm{~d} v$

The variation $\Delta$ is for the total time step $\mathrm{d} t$, the tension variation $\Delta \vec{\Phi}$, applied on the surface $S$, is obtained with the boundary conditions, and the final stress state will be deduced from the balanced stress variation.

\subsection{Constitutive equation}

The plastic response of the material is described using a classical Von Mises yield condition. The determination of the variation of the stress state is based on the normality principle of the plastic flow applied to a small strain variation:

$\delta \overline{\varepsilon^{\mathrm{p}}}=\lambda . \bar{n}$

where $\bar{n}$ is the unitary tensor normal to the yield stress surface:

$\bar{n}=\frac{3}{2} \cdot \frac{\bar{s}}{\sigma_{\text {eq }}}$

$\bar{s}$ is the deviatoric stress tensor, $\sigma_{\text {eq }}$ is the equivalent Von Mises stress and $\lambda$ is the equivalent plastic strain deduced from the projection of the tensorial elastoplastic constitutive equation onto the normal to the yield stress surface.

\subsection{Equilibrium equation solver}

\subsubsection{Solving algorithm}

To solve the equilibrium equation a constant operator strategy is used. The stiffness matrix of the problem is constituted with the elastic coefficients of the material and is integrated on the geometry at time $t$. Then the inverse stiffness matrix has to be computed only once per increment, which leads to shorter computation times. The total variation $\Delta$ for time step $\mathrm{d} t$ is cut into small iterative variations $\delta$, the equilibrium equation being written for an iteration $k$ in the following way:

$$
\begin{aligned}
& \int_{V}\left(\frac{1}{2} \bar{C}:\left(\operatorname{grad}^{T} \delta \vec{a}^{k}+\operatorname{grad} \delta \vec{a}^{k}\right)\right): \operatorname{grad} \vec{w} \mathrm{~d} v \\
& =\int_{S} \Delta \vec{\Phi}^{k} \cdot \vec{w} \mathrm{~d} s+\int_{V}\left(\bar{C}: \Delta \overline{\varepsilon^{\mathrm{p}} k}\right): \operatorname{grad} \vec{w} \mathrm{~d} v
\end{aligned}
$$

The second part of the equation is deduced from the variation of the solution at the previous iteration with the implicit integration of the constitutive law, the tension boundary at the surface $S$ being introduced only for the first iteration. The convergence of the resolution is achieved when the residual unbalance is lower than a very small scalar.

\subsubsection{Discrete decomposition}

A triangular finite-element with quadratic interpolation functions is used for the geometry and the displacement, the stress and the strain fields being linear on each element:

$\vec{a}^{k}=\vec{N}_{j} \cdot a_{j} \quad(j$ from 1 to 6$)$

The weak function is chosen as the displacement interpolation base, and the continuous equilibrium can be replaced in the approximation space by the following discrete system:

$K_{i j} . \delta a_{j}^{k}=\delta f_{i}^{k}$

$K_{i j}=\int_{V}\left(\frac{1}{2} \bar{C}:\left(\operatorname{grad}^{T} \vec{N}_{j}+\operatorname{grad} \vec{N}_{j}\right)\right): \operatorname{grad} \vec{N}_{i} \mathrm{~d} v$

$\delta f_{i}^{k}=\int_{S} \delta \vec{\Phi}^{k} \cdot \vec{N}_{i} \mathrm{~d} s+\int_{V}\left(\bar{C}: \delta \overline{\varepsilon^{\mathrm{p}} k}\right): \operatorname{grad} \vec{N}_{i} \mathrm{~d} v$

\subsection{Contact and friction law}

The contact and friction conditions are imposed with a penalty method. Eqs. (7)-(9) are written in a local coordinate system for all the concerned nodes. In order to keep a constant stiffness matrix in an increment of time $\mathrm{d} t$, the formulation must be explicit. If a boundary condition changes at iteration $k$ of increment $i$ the calculation is stopped. The matrix is then modified and the calculation restarts at the first iteration of increment $i$.

As the displacement of the tool $\delta \vec{a}_{0}$ is imposed, the unknown displacement is limited to a relative displacement of the node $\delta \vec{a}_{\mathrm{r}}$ so that Eq. (5) becomes:

$\left(K_{i j}-K_{f i j}\right) . \delta \vec{a}_{j}^{k}=\delta f_{j}^{k}$

with:

$$
\begin{aligned}
\delta f_{i}^{k}= & \int_{S} \delta \vec{\Phi}^{k} \cdot \vec{N}_{i} \mathrm{~d} s+\int_{S} \delta \tau_{\mathrm{f}} \frac{\delta \vec{a}_{0}}{\left\|\delta \vec{a}_{\mathrm{r}}\right\|} \cdot \vec{N}_{i} \mathrm{~d} s \\
& +\int_{V}\left(\bar{C}: \delta \overline{\varepsilon^{\mathrm{p}} k}\right): \operatorname{grad} \vec{N}_{i} \mathrm{~d} v \\
K_{f i j}= & -\int_{S} \delta \tau_{\mathrm{f}} \frac{1}{\left\|\delta \vec{a}_{\mathrm{r}}\right\|} \cdot \vec{N}_{i} \cdot \vec{N}_{j} \cdot \mathrm{d} s
\end{aligned}
$$


where $\tau_{\mathrm{f}}$ is the tangential friction stress.

The tool surfaces are modelled with linear elements on which different friction laws can be defined. The Tresca friction law, the Coulomb-Orowan friction law and the plastic wave model are available [11].

\subsection{Thermo-mechanical coupling}

\subsubsection{Energy-balance law}

Considering an axisymmetric domain $V$, the temperature field must satisfy the energy-balance equation:

$\rho . c \cdot \frac{\mathrm{d} T}{\mathrm{~d} t}=\operatorname{div} k \cdot \operatorname{grad} T+Q$

where $\rho$ is the density, $c$ is the heat capacity, $k$ is the heat conductivity, $T$ is the temperature and $Q$ is the internal source.

\subsubsection{Internal heat sources}

In the metal-forming case there are two kinds of internal sources that are deduced from the mechanical results: a fraction of the plastic strain power and the friction power at the workpiece-die interfaces.

\subsubsection{Prescribed boundary heat fluxes}

2.5.3.1. Convection. The prescribed boundary heat convection flux is a function of the heat transfer coefficient $\alpha(T)$ and the difference between the surface temperature $T$ and the room temperature $T_{\infty}$ :

$q=\alpha(T) .\left(T-T_{\infty}\right)$

2.5.3.2. Radiation. An evolutive closed-cavity model with current view factors is used [12].

Radiation emission: each point along the boundary of a cavity $C$ emits a flux $\phi$ proportional to the temperature to the fourth power, to the Stefan-Boltzmann constant $\sigma$ and to the surface emissivity $\varepsilon$ :

$\phi=\varepsilon . \sigma . T^{4}$

Absorption-reflection: according to the Kirchoff law, the absorption and reflection coefficients are equal in the same direction and frequency. Extending this law to every direction of space and every frequency of the signal spectrum:

$\phi_{\mathrm{a}}=\varepsilon \phi_{\mathrm{i}}$ and $\phi_{\mathrm{r}}=(1-\varepsilon) \phi_{\mathrm{i}}$

where $\phi_{\mathrm{i}}$ is the incident flux, $\phi_{\mathrm{a}}$ is the absorbed flux and $\phi_{\mathrm{r}}$ is the reflected flux.

\subsection{Stress state in the tools}

\subsubsection{Hypothesis}

As the strains are supposed small, the tool meshes remain constant from the start to the end of the simula- tion, and are totally independent of the thermal meshes. The radial displacements of the tool nodes that are at the press boundary are usually free but can also be stopped.

\subsubsection{Solving algorithm}

The discrete decomposition is also a triangular finite element composed of six nodes and three integration points and the solving algorithm used to find the tool stress state is similar to that described for the workpiece.

When the strain and the stress states are known for each Gauss point of the workpiece, they are extrapolated at the tool-workpiece interface nodes. As the tool border nodes are different from those of workpiece, the normal stress and the tangential stress are interpolated linearly and used as the loading conditions for the tool-solving algorithm.

\section{Failure-prediction routine}

\subsection{Brittle fracture modelling}

The statistical nature of the fracture of ceramics is connected to the presence of flaws in the material. Indeed, the failure of ceramic structure depends on the size, on the orientation and on the position in the structure of the critical flaw which initiates the fracture. Because this flaws distribution is impossible to determine, some statistical functions are used to describe the strength variability of a structure.

In his theory, Weibull associates a strength $\sigma_{\mathrm{c}}$ with each elementary volume $V_{\mathrm{e}}$. The strength of the material is characterised by the distribution function of $\sigma_{\mathrm{c}}$, denoted $F(\sigma)$, which is defined as the number of all elementary volumes having $\sigma_{\mathrm{c}} \leq \sigma$, divided by the total volume. This function also gives the probability $P$ of randomly choosing an elementary volume having a strength equal to or less than $\sigma$, or also the probability that this volume fails under the stress $\sigma$ :

$P\left(\sigma_{\mathrm{c}} \leq \sigma\right)=F(\sigma)$

Any distribution function may be written in the form:

$F(\sigma)=1-\exp (-\phi(\sigma))$

where $\phi(\sigma)$ must be a positive, non-decreasing function, vanishing at a value $\sigma_{\mathrm{u}}$, which is not necessarily equal to zero.

The advantage of this equation appears when the failure probability of the structure constituted by all the elementary volumes, using the weakest link theory, is considered. If it is supposed that the total volume $V$ fails if any one of its parts fails, then the probability of non failure, under an uniform uniaxial load $\sigma$, is equal to: 
$(1-P)^{n}=\exp (-n \phi(\sigma))$

where $n$ is the number of elementary volumes of the structure $\left(V=n V_{\mathrm{e}}\right)$.

The failure probability $P_{n}$ of the structure takes then the simple form:

$P_{n}=1-\exp (-n \phi(\sigma))$

Weibull has chosen a power form for the function $\phi(\sigma)$, which is the most simple function satisfying the general conditions previously described:

$\phi(\sigma)=\left(\frac{\sigma-\sigma_{\mathrm{u}}}{\sigma_{0}}\right)^{m}$

where $\sigma_{\mathrm{u}}, \sigma_{0}$ and $m$ are parameters depending on the material characteristics.

The distribution function obtained has no theoretical basis, but experience has shown that in many cases it gives a good description of the strength fracture reliability of brittle materials. The threshold stress $\sigma_{\mathrm{u}}$ is usually taken as equal to zero. The two other parameters can then be identified easily by comparison with results of fracture tests.

In the case of non uniform uniaxial load $\sigma$, the failure probability takes the integral form:

$P=1-\exp \left(-\frac{1}{V_{\mathrm{e}}} \int_{V}\left(\frac{\sigma}{\sigma_{0}}\right)^{m} \mathrm{~d} V\right)$

This expression can be generalised to multi-axial stress states. Two strategies are possible: the first consists in considering an equivalent stress that characterises the loading at each point; the second consists in supposing the independence of action of each principal tensile stress [2].

Weibull, taking up the first approach, proposed to calculate an equivalent stress by averaging the normal tensile stress in all directions. This model leads to a complex integral formulation, which may increase the computation time. In order to obtain shorter computation times, only the maximal normal stress has been considered in the present model, as has been done by other investigators [4]. This variable is also the maximal principal stress at each point of the structure.

In the second approach, the fracture probability depends on all of the tensile principal stresses. The independence of action of the principal stresses means that reliability of the structure is the product of the reliability of the individual principal stresses acting independently. The fracture probability is then given by the equation:

$P=1-\exp \left(-\frac{1}{V_{\mathrm{e}}} \int_{V}\left(\left(\frac{\sigma_{1}}{\sigma_{0}}\right)^{m}+\left(\frac{\sigma_{2}}{\sigma_{0}}\right)^{m}+\left(\frac{\sigma_{3}}{\sigma_{0}}\right)^{m}\right) \mathrm{d} V\right)$

where $\sigma_{1}, \sigma_{2}$ and $\sigma_{3}$ are the positive principal stresses or take the zero value for the compressive principal stresses.
It is interesting to note that in the both of the retained models, the compressive stresses are not taken into account, it being supposed that fracture is always the result of tensile stresses. The present authors have made this choice because the compressive fracture strength of ceramics is three or four times higher than the tensile strength, so that it is very unlikely that a structure would fail under compressive stresses.

\subsection{Numerical introduction of the model}

Both models previously described have been introduced into POLLUX. The architecture and basic computational element of the failure prediction program are described in Fig. 1. First, the thermomechanical analysis is achieved, leading to the calculation of the stress tensor on each point of the tools, which latter are supposed to be perfectly elastic. Then, the failure probabilities of the tools are determined, using the Weibull parameters and criteria chosen by the user.

The discrete decomposition and the Gauss quadrature of the models gives, if $V_{\mathrm{e}}$ is arbitrary taken equal to unity:

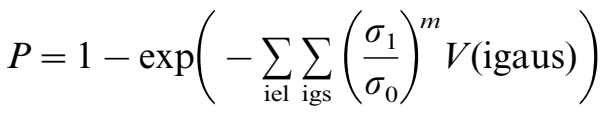

for the maximal principal stress criteria (criteria 1), and:

$$
\begin{aligned}
& P=1-\exp \left(-\sum_{\text {iel }} \sum_{\text {igs }}\left(\left(\frac{\sigma_{1}}{\sigma_{0}}\right)^{m}+\left(\frac{\sigma_{2}}{\sigma_{0}}\right)^{m}+\left(\frac{\sigma_{3}}{\sigma_{0}}\right)^{m}\right)\right. \\
& V \text { (igaus)) }
\end{aligned}
$$

for the principle of independent actions (criteria 2), in which: $\sigma_{1}, \sigma_{2}, \sigma_{3}$ are the principal stresses in the Gauss point igaus of element iel $\left(\sigma_{1} \geq \sigma_{2} \geq \sigma_{3}\right)$ or equal to zero for compressive principal stress, where $V$ (igaus) is the volume associated to the Gauss point igaus.

The elementary failure probability DPr, or local risk of rupture, is also calculated at each Gauss point, and interpolated to the nodes (Fig. 1). This variable corresponds to the fracture probability of an elementary volume of constant size equal to unity, supporting the stress state of the point considered. The values can be stored in a data file for graphical display of the structure critical regions. Another data file, containing the failure probability of overall structure as a function of time or tools displacement, is also generated. At the end of each increment, the program compares the failure probability of the tools to the maximal probability permitted, chosen by the user. The computation is stopped when this limit or the maximal displacement of the tools is achieved. 


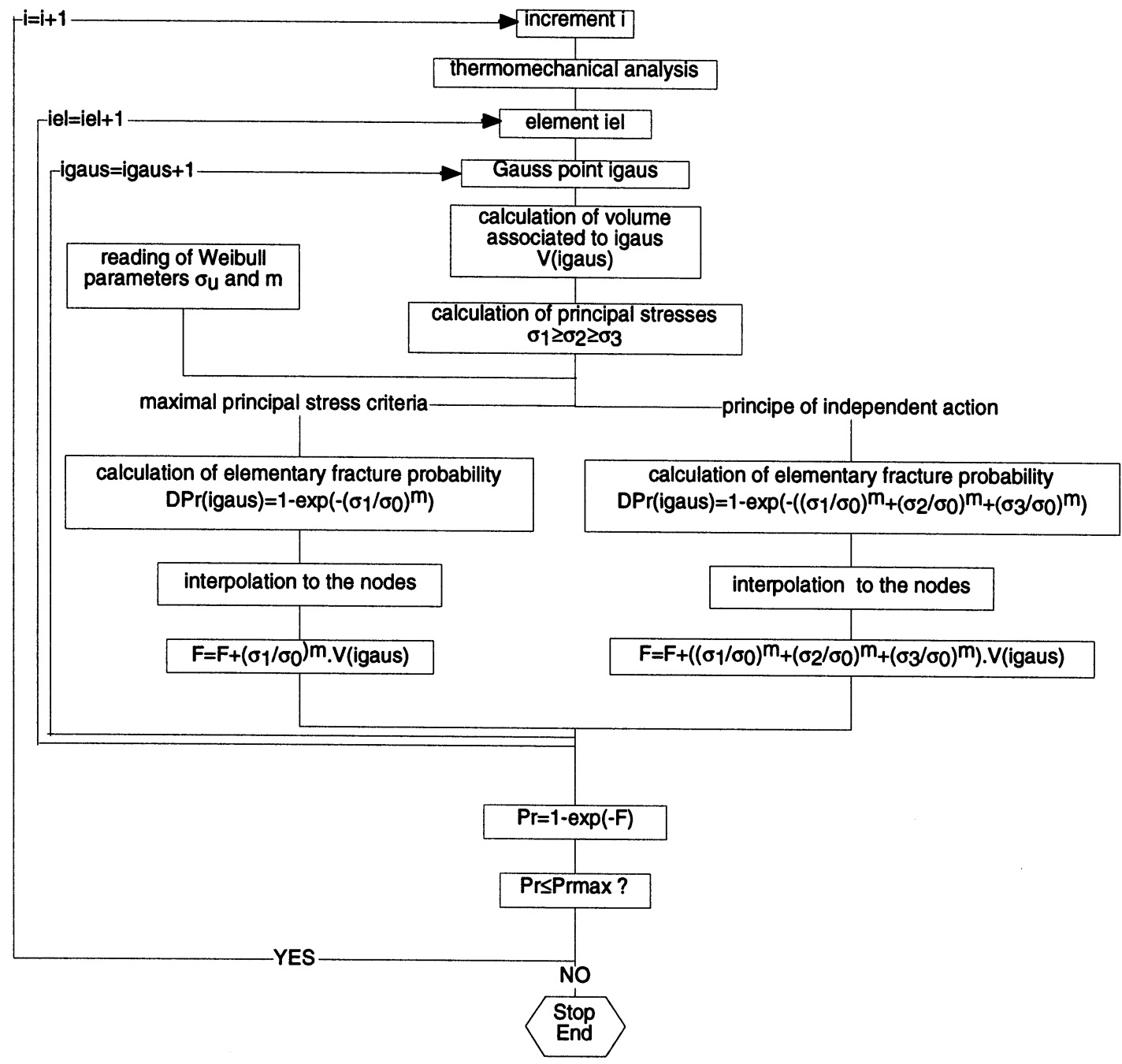

Fig. 1. Computational elements of the failure-prediction routine.

\section{Model testing}

In order to assess the validity of the numerical model developed, the software has been tested for the case of a ceramic cylinder under compression loading, considering an elastic cylindrical workpiece of radius $R$ and

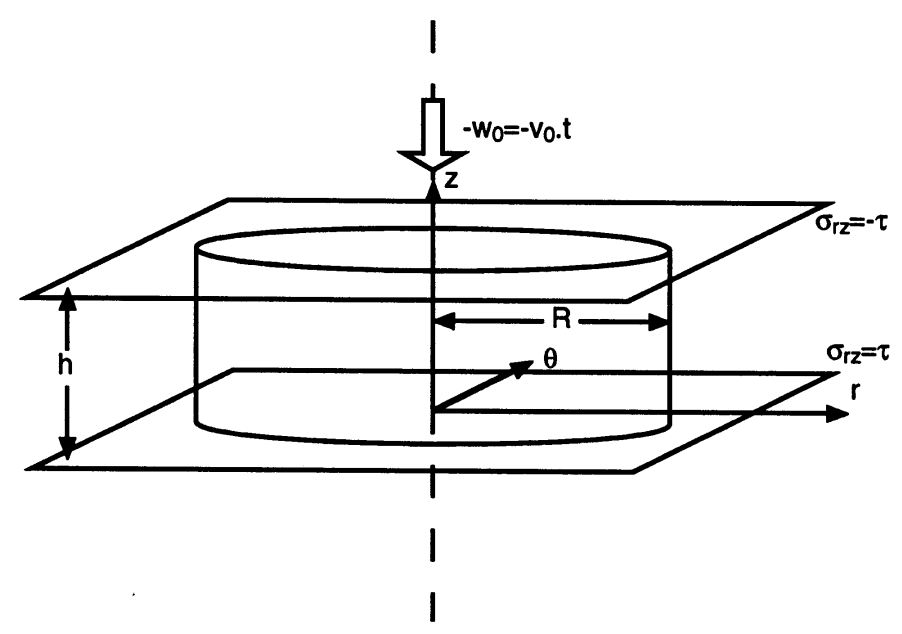

Fig. 2. A cylindrical workpiece pressed between two planes with uniform friction. height $h$, between two vertical planes (Fig. 2). A constant velocity $v_{0}$ is prescribed on the upper plane, whilst the lower plane has no displacement in the vertical direction. The shear stress $\tau(\tau>0)$ between the planes and the workpiece is assumed to be constant, except for near to the symmetry axis, where it increases in a linear manner with the radius $r$.

The failure probability of the cylinder, computed by the software as a function of the tool displacement, has been compared to the results of an analytical calculation. For this validation, the following changes have been provided to the failure prediction program: the failure prediction calculation is run in the workpiece instead of in the tools; the stress state in the cylinder being essentially compressive, the absolute value of the principal stress has been considered for the calculation of the failure probability.

\subsection{Analytical calculation}

\subsubsection{Basic hypothesis}

It is supposed that the flow is axisymmetric, and that the axial displacement is independent of $r$ : 


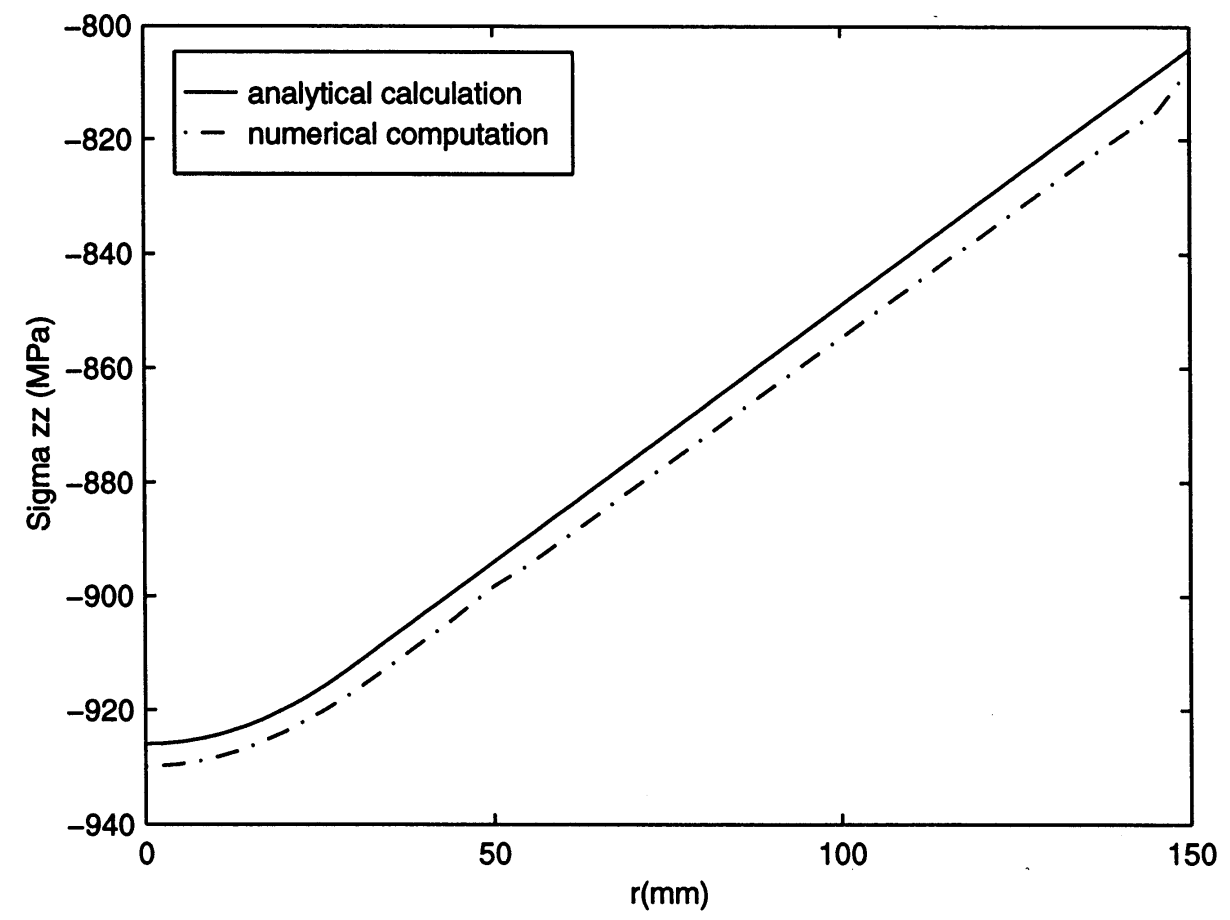

Fig. 3. Evolution of the stress $\sigma_{\mathrm{zz}}$ as a function of the radius $r$.

$w(z)$

\subsubsection{Boundary conditions}

According to the problem description (Fig. 2), boundary conditions (in local coordinates) are:

$z=0 \quad\left\{\begin{array}{cc}w=0 & \\ 0 \leq r \leq \varepsilon & \sigma_{r z}=-\underset{\varepsilon}{\varepsilon} \\ \varepsilon \leq r \leq R & \sigma_{r z}=-\tau\end{array}\right.$

$z=h \quad \begin{cases}w=-w_{0}=-v_{0} . t & \\ 0 \leq r \leq \varepsilon & \sigma_{r z}=\underset{\varepsilon}{r} \\ \varepsilon \leq r \leq R & \sigma_{r z}=\tau\end{cases}$

$r=R \quad \sigma_{r r}=0$

\subsubsection{Solution}

The resolution of the average equilibrium equations, using the average of the values of the stresses and of the radial displacement, gives the following expressions for the average stress $\frac{\text { g }}{\sigma_{z z}}$ :

$$
\begin{aligned}
0 \leq & r \leq \varepsilon \\
& \overline{\sigma_{z z}}(r) \\
= & \frac{v}{(1-v)} \frac{\tau}{h}\left(\frac{r^{2}}{\varepsilon}+\varepsilon+\frac{(1-2 v)}{6} \frac{\varepsilon^{3}}{R^{2}}-\frac{4}{3}(2-v) R\right)-\frac{E w_{0}}{h}
\end{aligned}
$$

$$
\begin{aligned}
\varepsilon \leq & \overline{ }\left(\overline{\sigma_{z z}}(r)\right. \\
= & \frac{v}{(1-v)} \frac{\tau}{h}\left(2 r+\frac{(1-2 v)}{6} \frac{\varepsilon^{3}}{R^{2}}-\frac{4}{3}(2-v) R\right)-\frac{E w_{0}}{h}
\end{aligned}
$$

if $(h / R) \ll 1$, it is assumed that:

$\bar{\sigma}(r, z) \cong \bar{\sigma}(r)$

In addition, if $\tau \ll \sigma_{\mathrm{rr}}, \sigma_{\theta \theta}, \sigma_{\mathrm{zz}}$, then $\sigma_{\mathrm{rr}}, \sigma_{\theta \theta}$ and $\sigma_{\mathrm{zz}}$ can be considered as the principal stresses at each point of the cylinder, and $\sigma_{\mathrm{zz}}$ as the maximal principal stress. The failure probability of the cylinder can then be expressed, using the criteria 1 as:

$P=1-\exp \left(-2 \pi h \int_{0}^{R}\left(\frac{\left|\overline{\sigma_{z z}}(r)\right|}{\sigma_{0}}\right)^{m} r \mathrm{~d} r\right)$

The value of $\varepsilon$ is obtained by identification with the stress field given by numerical computation.

The integral

$\left(\int_{0}^{R}\left(\frac{\left|\overline{\sigma_{z z}}(r)\right|}{\sigma_{0}}\right)^{m} r \mathrm{~d} r\right)$

having no analytical solution, has been evaluated numerically, for different values of $w_{0}$, using an adaptive recursive Simpson rule [13].

\subsection{Numerical results}

Analytical and numerical values of the stress $\sigma_{\mathrm{zz}}$ as a function of radius $r$ are compared on Fig. 3, for a displacement of the upper plane $w_{0}$ equal to $0.03 \mathrm{~mm}$. 


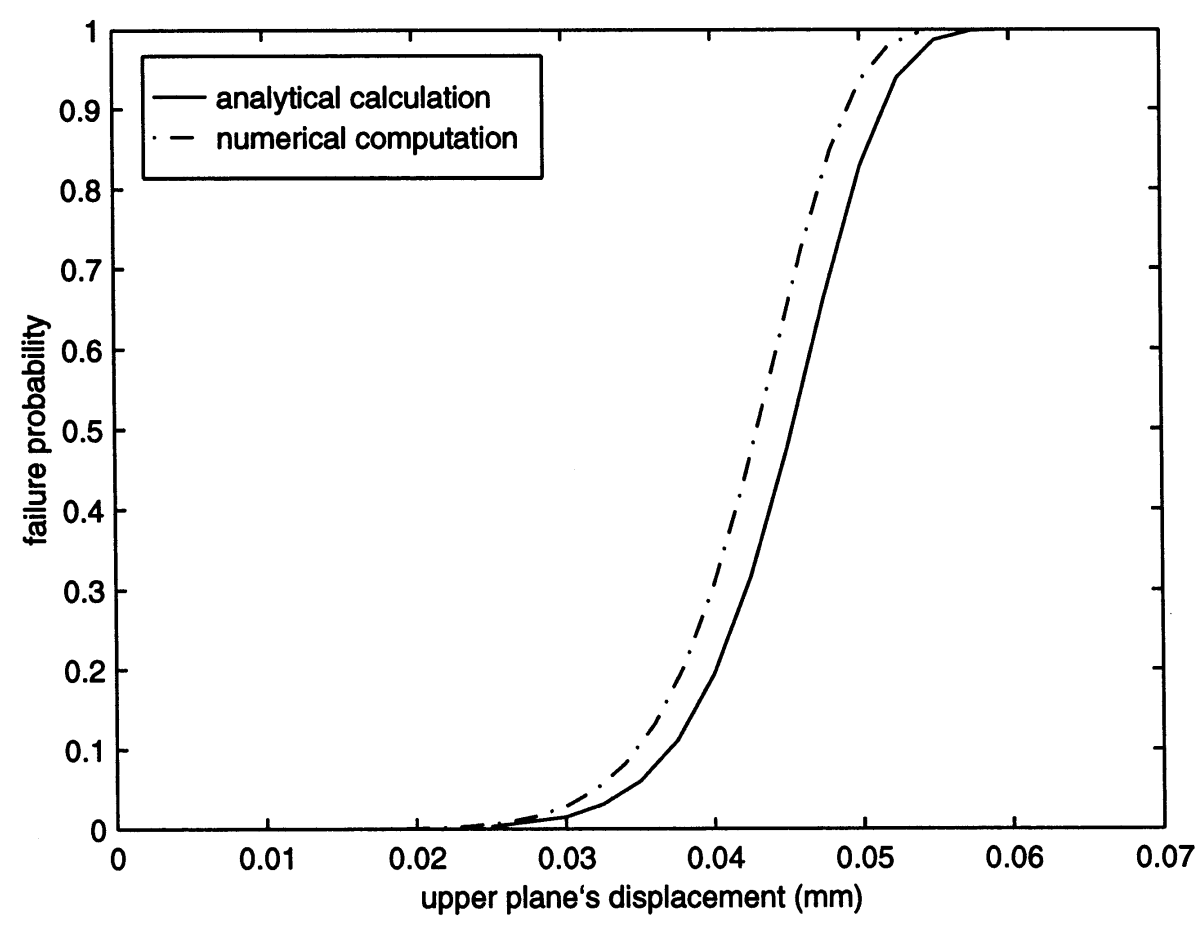

Fig. 4. Evolution of the failure probability of the cylinder as a function of the displacement of the upper tool.

The analytical calculation gives a slightly lower stress $(1 \%)$ than that of the numerical computation, but nevertheless it gives a good approximation.

Fig. 4 represents the evolution of failure probability calculated with the finite-element program (criteria 1), and with the analytical expression for the stress $\overline{\sigma_{z z}}$. The two curves are relatively close, nevertheless, the analytical calculation give significantly lower values than the numerical computation, which is in accordance with the difference observed in the stress values and with the high value of the $m$ parameter $(m=12)$.

\section{Application: design of ceramic forging dies}

The software has been developed in order to be able to predict the reliability of ceramic tools during a forging operation, the resulting information enabling the improvement of the design of the tools.

Two data types are accessible: (i) the overall failure probability of the tool as a function of its displacement, or of time, the reliability of the tool during one operation being deduced from the maximal value of this probability; (ii) the elementary failure probability, or local risk of rupture at a given time of the forging process, which allows the identification of the tool critical regions.

These two items of information are required for the designing of the tools. The design technique using failure prediction software consists of three steps: in the first step, the user defines the tool initial geometry and reliability, according to the type and the geometry of the forged piece; in the second step, the software calcu- lates the overall failure probability of the tools, using the initial geometry, where if the corresponding reliability is consistent with the defined reliability, then the geometry is convenient. On the contrary, if the calculated reliability is lower than the chosen reliability, then the third step consists in modifying the geometry of the tools, according to the local risk of rupture. These data enable the user to identify the critical zones of the tools which must be modified. It is then possible, by local change in the tool geometry, to increase the reliability without modifying in a significant manner the final shape of the forged piece.

This technique has been applied to a simple case of isothermal forging. The workpiece material is of Astroloy (nickel-base alloy) and the forging dies are of refractory ceramic.

\subsection{Forging modelling}

The running of the POLLUX code with the failure prediction program requires the creation of three files. The first file contains the geometry of the initial workpiece and of the tools. As described earlier, the first step in the design method must be the definition of the tool initial geometry, according to the shape of the final piece. The second file contains the data of the mechanical and thermal behaviour of the different materials, the initial and boundary conditions (temperature, tools displacement, etc.), and the friction conditions between the workpiece and the tools. The third file contains the fracture parameters of the tools material. The following sections present the data contained in these different files. 


\subsubsection{Geometry}

For this example, a simple geometry has been chosen for the final piece (Fig. 5). According to this shape, the tools geometry has been defined (Fig. 6). The lower tool is flat with an axisymmetric geometry, like the final piece, whilst the engraving of the upper tool corresponds to the shape of the piece to be obtained. The radius in the concave fillet of the engraving was not indicated in the geometry of the piece, and has been taken as $3 \mathrm{~mm}$. The
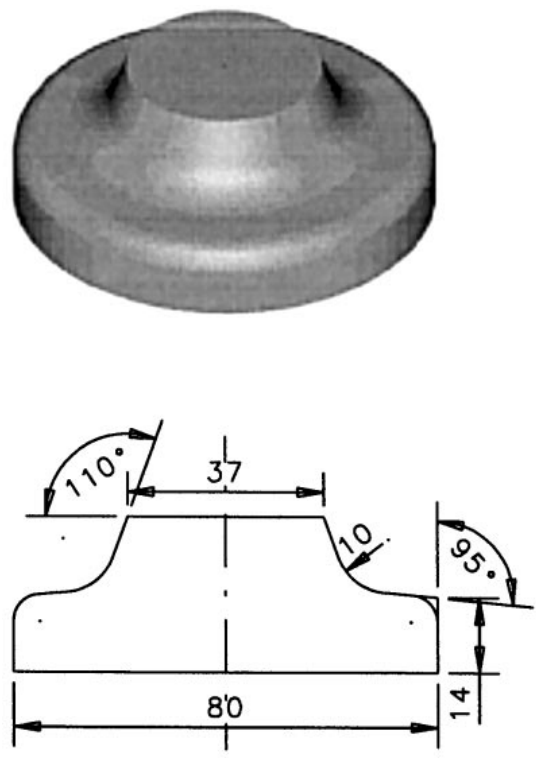

Fig. 5. Final geometry of the forged piece (dimensions: $\mathrm{mm}$ ).
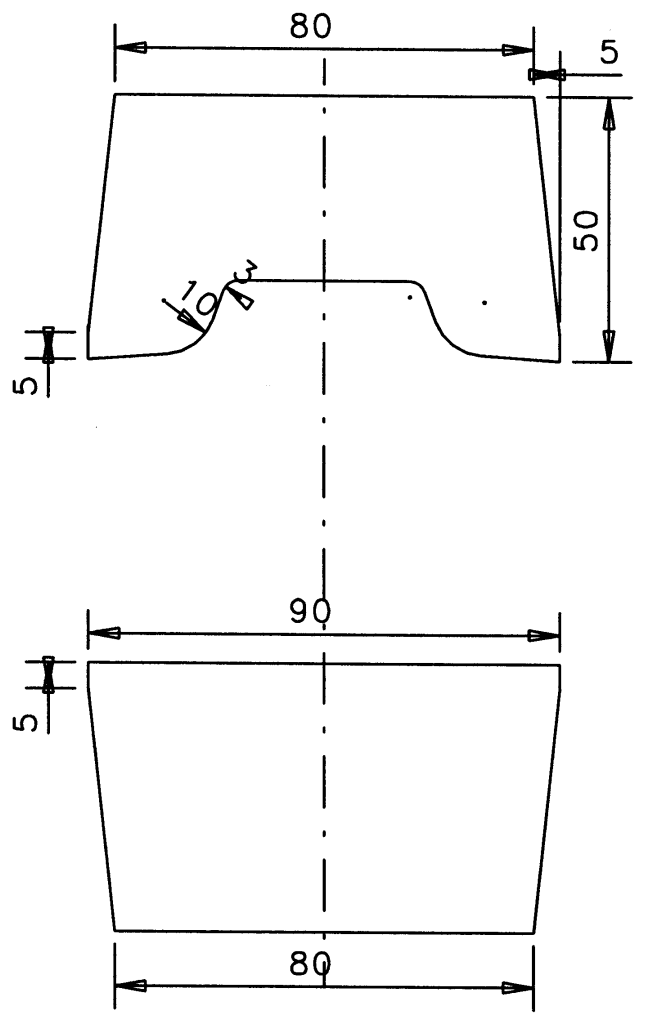

Fig. 6. Geometry of the tools (dimensions: $\mathrm{mm}$ ).
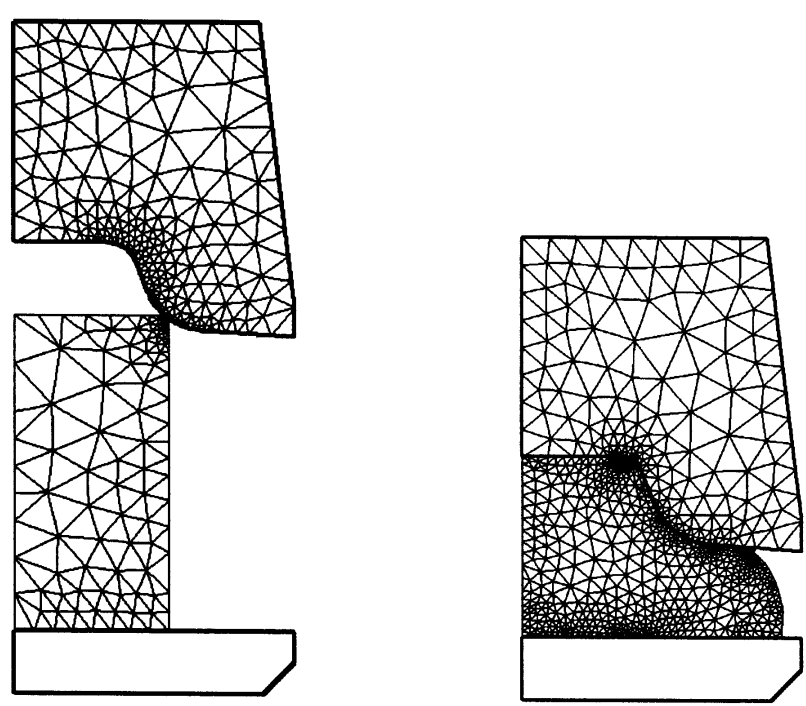

Fig. 7. Initial and final configuration of the finite-element simulation.

workpiece has a cylindrical initial geometry, with a height of $50 \mathrm{~mm}$ and a diameter of $50 \mathrm{~mm}$.

\subsubsection{Material behaviour and initial and boundary conditions}

Astroloy material has superplastic behaviour at temperatures above $1323 \mathrm{~K}$, and at low strain rates. The simulation of an isothermal forging operation at the temperature of $1373 \mathrm{~K}$, with a downwards speed of the upper tool of $0.1 \mathrm{~mm} \mathrm{~min}^{-1}$ (the lower tool remains fixed), has been chosen. Heat transfer can be neglected, the temperature of the tools and the workpiece being assumed to be constant during the operation.

The mechanical behaviour of the tools is supposed to be perfectly elastic during the forging operation. Young's modulus and the Poisson coefficient are respectively equal to $262 \mathrm{GPa}$ and 0.3 at the forging temperature.

The rheological behaviour of the workpiece is assumed to be viscoplastic in the process range temperature. A constitutive model has been proposed by Soucail for Astroloy material [14]:

$\bar{\sigma}=K(T) \cdot \dot{\bar{\varepsilon}}^{n}$

where $\bar{\sigma}$ is the Von Mises equivalent stress (MPa); $\dot{\bar{\varepsilon}}$ is the equivalent strain rate $\left(\mathrm{s}^{-1}\right) ; K(T)$ is a proportionality constant, taken as equal to 119 at $1373 \mathrm{~K}$; and $n$ is the strain-rate sensitivity factor, taken as equal to 0.19 . Friction between the tools and the workpiece is modelled by the Coulomb law, with a coefficient $\mu$ of 0.1 .

\subsubsection{Fracture parameters of material tools}

The Weibull parameters describing the fracture characteristics of the used ceramic have been determined according to the results of four point bending tests, run at $1373 \mathrm{~K}$. The $\sigma_{0}$ and $m$ values are respectively equal to $400 \mathrm{MPa}$ and 12 , and are assumed to be constant during the whole of the forging process. 


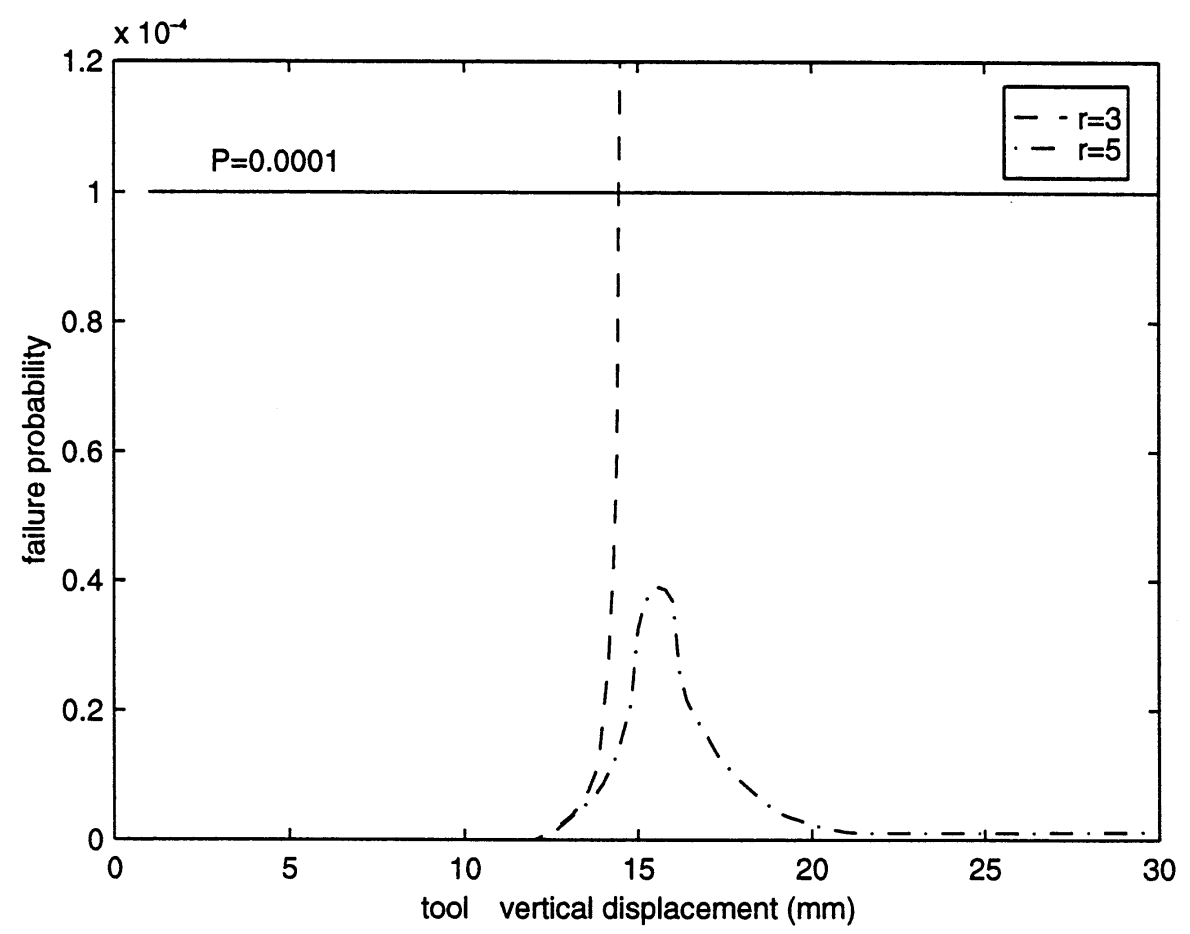

Fig. 8. Evolution of the fracture probability of the upper tool as a function of its displacement, for two different engraving concave fillet radii.

These data have been deduced from quasi-uniaxial stress-state tests, which makes it difficult to choose the multi-axial stress-state criteria. Thus, it is assumed arbitrarily that the maximal principal stress criteria (criteria 1) is more suitable for the present ceramic material.

A tool reliability of 0.9999 has been chosen, which means that the failure probability of the tools must be always lower than $10^{-4}$ during the process cycle.

\subsection{Failure prediction results}

To obtain a forging piece with a near net shape, the final displacement of the upper tool has been fixed at 30 $\mathrm{mm}$. The initial and final configurations of the simulation computation are presented in Fig. 7.

Owing to its being flat, the lower tool is subjected to essentially only compressive stresses, so that its failure probability is very low compared to that of the upper tool. In the latter, the engraving leads to the occurrence of stress concentration during forging, which increases the failure probability. Fracture-prediction software has thus been used for the upper tool only.

The evolution of the overall fracture probability of the upper tool is represented in Fig. $8(r=3)$ as a function of the vertical displacement of the tool. This probability increases swiftly, and overshoots the fixed limit before the end of the forging operation. The stress state to which the upper tool is subjected is too severe to preserve the fixed reliability. The tool geometry must be then modified to improve the reliability, according to indications given by the local risk of rupture distribution (Fig. 9).
The graphical analysis of local failure probability allows the identification of regions sustaining the more critical stress states. According to this distribution, the maximal local risk is localised around the bottom radius of the engraving. The stress concentration in this zone is too high.

The stress field around a corner being related to the value of its radius, the increasing of the bottom radius of the engraving leads to a decrease of the stress concentration factor, and correspondingly to a decrease of failure probability of the piece.

A second tool geometry can then be defined, with a radius in the concave fillet engraving of $5 \mathrm{~mm}$ instead of $3 \mathrm{~mm}$. The overall fracture probability corresponding to this new geometry is represented as a function of tool displacement in Fig. $8(r=5)$ : the global risk does not exceed $10^{-4}$, which is in agreement with the fixed reliability.
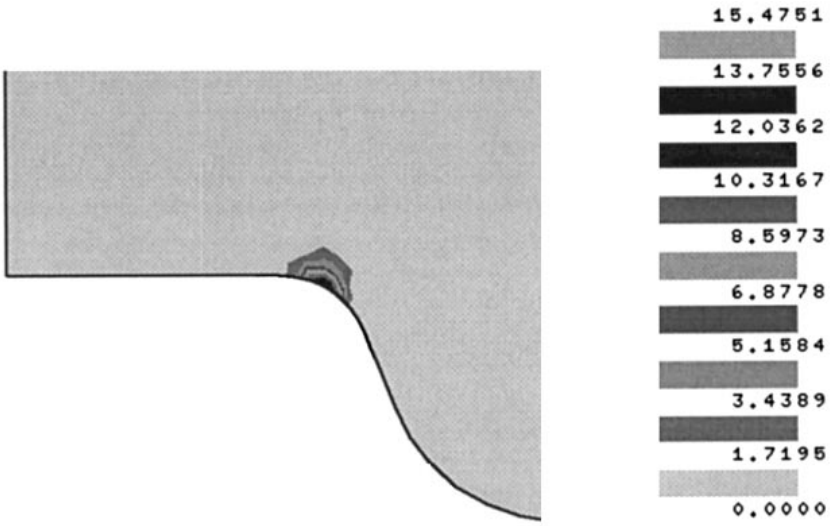

Fig. 9. Local risk of rupture (\%) of the upper tool around the engraving fillet radius. 


\section{Conclusions}

A fracture-prediction software for brittle materials has been introduced into the POLLUX finite-element code, in order to be able to predict the risk of rupture of ceramic tools during a forging operation. Comparison between the code results and the results of the analytical calculation in a simple compression case has led to the validation of the implementation of the numerical model. The use of this software allows the improvement of the design of ceramic forging tools. The utilisation strategy is presented for a forging example of a simple shape piece.

The fracture-prediction software requires, however, the knowledge of experimental data about the fracture behaviour of the ceramic material used. These data enable the choice of the failure criteria that is more convenient for the material, and the identification model parameters. Two failure criteria are already available. Future developments forecast the introduction of other criteria, including shear stresses, and the differentiation between two types of defects (surface defaults and volume defects, for instance). In addition, fracture prediction tests are currently being performed on computer-designed samples in order to further validate the chosen numerical model.

\section{Acknowledgements}

The ceramic material was provided by Norton Desmarquest (Evreux F27), the help of Mr Cales and $\mathrm{Mr}$ Main being gratefully acknowledged. Information on POLLUX software can be supplied by Dr J.C. Boyer.

\section{References}

[1] W.A. Weibull, A statistical distribution function of wide appli- cability, J. Appl. Mech. 18 (3) (1951) 293-297.

[2] A.M. Freudenthal, Statistical approach to brittle fracture, in: $\mathrm{H}$. Liebowitz (Ed.), Fracture, vol. 2, An Advanced Treatise, Mathematical Fundamentals, Academic Press, New York, 1968, pp. $591-619$.

[3] S.B. Batdorf, J.G. Crose, A statistical theory for the fracture of brittle structures subjected to nonuniform polyaxial stresses, J. Appl. Mech. 41 (2) (1974) 459-464.

[4] C. Berdin, Etude expérimentale et numérique de la rupture des matériaux fragiles, Thèse de doctorat de l'Ecole des Mines de Paris, Paris, 1993.

[5] L. Guillaumat, J. Lamon, Simulation statistique-probabiliste du comportement mécanique non-linéaire d'un composite 2D SiC/ $\mathrm{SiC}$, in: Proceedings Journée Nationale sur les Composites, vol. 2, Saint-Etienne, France, 1994.

[6] J.P. Gyekenyesi, SCARE: a postprocessor program to MSC/ NASTRAN for reliability analysis of structural ceramic components, J. Eng. Gas Turbines Power 108 (1986) 540-546.

[7] J.P. Gyekenyesi, N.N. Nemeth, Surface flaw reliability analysis of ceramic components with the SCARE finite element postprocessor program, J. Eng. Gas Turbines Power 109 (1987) 274281.

[8] N.N. Nemeth, L.M. Powers, L.A. Janosik, J.P. Gyekenyesi, Designing ceramic components for durability, Am. Ceram. Soc. Bull. 72 (12) (1993) 59-69.

[9] N.N. Nemeth, J.M. Manderscheid, J.P. Gyekenyesi, Designing ceramic components with the CARES computer program, Ceram. Bull. 68 (2) (1989) 2064-2072.

[10] B. Michel, J.C. Boyer, Elasto-visco-plastic finite element analysis of a cold upsetting test and stress state validation by residual stress measurements, Adv. Mater. Process. Technol. 3 (1993) $1701-1717$.

[11] L. Baillet, J.C. Boyer, A friction model for closed-die forging FEM simulation, in: Metal Forming Process Simulation in Industry, International Conference and Workshop, vol. 1, 28-30 September 1994, Baden-Baden, Germany, 1994, pp. 132-145.

[12] C. Garot, P. Gendre, Computation of view factors used in radiant energy exchanges in axisymetric geometry, in: P.W. Lewis, K. Morgan (Eds.), Numerical Methods in Thermal Problems, Pineridge Press, Swansea, UK, 1979, pp. 99-108.

[13] G.E. Forsythe, M.A. Malcolm, C.B. Moler, Computer Methods for Mathematical Computations, Prentice-Hall, Englewood Cliffs, NJ, 1977.

[14] M. Soucail, L. Naze, A. Moal, J.P. Ferte, Modélisation du soudage par friction inertielle du superalliage à base de nickel astroloy, Rev. Sci. Snecma 4 (1993) 33-44. 